\title{
La relación médico-paciente en los tiempos del SIDA: el casopsicoanalítico
}

\author{
Mark Platts
}

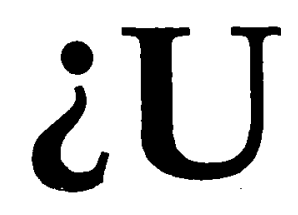

\section{na nueva relación médico-paciente?}

Todo paciente, como todo médico, tiene sus individualidades; esto, aunado a la gran variedad de estructuras e instituciones en donde se ubican las relaciones médico-paciente, da lugar a una notable diversidad dentro de esa relación. Con todo, un médico norteamericano, Mark Siegler, ha desarrollado un modelo general de la relación médico-paciente que nos permite entender mejor el marco general dentro del cual suceden tantas variaciones. ${ }^{1}$ En este modelo hay "cuatro momentos" o etapas: (1) la fase pre-paciente; (2) el proceso de reunir y reducir los datos pertinentes; (3) la etapa de acomodo entre el paciente y el médico; y (4) el establecimiento de la relación plena médico-paciente. Desde luego, no toda la gente ejemplifica en la realidad esta sencilla secuencia temporal; por diversas razones, una relación específica médico-paciente podría saltar una o más de las etapas distinguidas y por otras razones una persona podría moverse de una fase a otra y luego volver a la fase anterior.

En la fase pre-paciente una persona se da cuenta -0 cree que se da cuenta- de que algo anda mal en su vida en relación con sus expectativas. Cómo conceptualizamos tal experiencia, y cómo reaccionamos frente a tal experiencia, está influido por diversos factores psicológicos, sociales, culturales y económicos. Una opción evidente es la de conceptualizar el malestar como una posible manifestación de algún problema médico y la de reaccionar recurriendo a una consulta médica. Probablemente esta opción estará motivada por la esperanza de encontrar algún tratamiento médico eficaz en

'Mark Siegler, "The nature and limits of clinical medicine", en E. J. Cassel y M. Siegler (comps.), Changing Values in Medicine. Bethesda, Md., 1979, pp. 1941. 
relación con las fuentes de su malestar, pero también podría estar motivada por el deseo de lograr la legitimación de su papel de enfermo, tal y como lo describe Talcott Parsons, ${ }^{2}$ un deseo generalmente determinado, en parte, por diversas presiones provenientes de la conjunción de relaciones personales y laborales. El uso del concepto de enfermedad no está restringido exclusivamente a contextos específicamente médicos.

Una vez ya frente al médico, se inicia el proceso de reunir y reducir los datos pertinentes con el propósito de construir un diagnóstico del problema del paciente. Tanto la forma de conceptualizar el supuesto problema como la forma de manejarlo una vez que se haya emitido el diagnóstico, dependerá en parte del tipo de médico involucrado: un gastroenterólogo no es un psicoanalista, un homeópata no es un terapeuta conductista. De este modo se inicia la tercera etapa, la del acomodo entre el paciente y el médico. El diagnóstico ha sido que el paciente tiene algún problema médico $y$, por lo tanto, necesita la ayuda de algún médico: el propósito del proceso de acomodo es el de determinar si este médico habrá de ser el médico de este paciente. Hay consideraciones muy obvias -algunas razonables, otras no tanto- que pueden hacer que el paciente prefiera buscar otro médico; hay consideraciones tal vez menos obvias que pueden hacer que el médico decida no aceptar al paciente como su paciente. Pero si tales consideraciones no entran en juego, y si el proceso de acomodo sigue sin problemas mayores podría llegarse a la última etapa, el establecimiento de la relación plena médico-paciente. El meollo de tal relación es lo que el doctor Laín Entralgo llamó la philia médica, ${ }^{3}$ una firme amistad médica basada en la existencia de un profundo vínculo de confianza entre el paciente y el médico.

A pesar de lo esquemática que ha sido esta presentación del modelo de Siegler, nos permite reconocer algunas de las posibles dificultades distintivas que podrían surgir en las relaciones entre médicos y pacientes en los casos en donde: (i) el paciente está infectado por el virus de la inmunodeficiencia humana; (ii) la forma de su infección ha sido vía relaciones homosexuales; y (iii) el médico a quien recurre para buscar ayuda es un psicoanalista. Se trata, pues, de un paciente que pertenece a un grupo que tradicionalmente ha sido severamente discriminado y que sigue siendo el objeto de poderosos prejuicios, tanto por parte de la sociedad en general como por parte de familiares, colegas, patrones y trabajadores de la salud. Se trata de alguien que, en con-

2 Talcott Parsons, The Social System. Nueva York, 1951 y "Definitions of health and illness in the light of American values and social structure", en E. G. Jaco (comp.), Patients, Physicians and Illness: A Sourcebook in Behavioural Science and Health. Nueva York, 1972, pp. 107-127.

${ }^{3}$ Pedro Lain Entralgo, Doctor and Patient, trad. F. Partridge. Nueva York, 1969. 
secuencia, padece una condición médica altamente estigmatizada en relación con la cual existen niveles horrorosos de ignorancia y mitología, de nuevo tanto por parte de la sociedad en general como por parte de familiares, colegas, patrones y trabajadores de la salud. ${ }^{4}$ Se trata, además, de una condición médica con una muy alta probabilidad de conducir a una enfermedad, el SIDA, que además de ser objeto de las mismas actitudes negativas mencionadas respecto de la condición de seropositividad en relación con el viH, es también mortal. Se trata, finalmente, de una específica combinación de circunstancias -homosexualidad más seropositividad-que ha recibido poca, pero muy poca, atención por parte de los psicoanalistas.

Sería sorprendente que una persona como la descrita al encontrarse en la fase pre-paciente contemplara la posibilidad de recurrir a un psicoanalista en búsqueda de una cura de su condición, sea en lo referente a su homosexualidad o a su seropositividad. Pero también sería sorprendente que tal persona contemplara esa posibilidad motivada por un deseo de conseguir la legitimación de su papel de enfermo: la penumbra perniciosa que resulta de la mezcla de discriminación, prejuicio, estigmatización, ignorancia y mitología, debería desalentar cualquier pensamiento en ese sentido. ${ }^{5}$ Más bien, su motivación para contemplar la posibilidad de recurrir a un psicoanalista habrá de ser la de aliviar algunos de los efectos secundarios de su condición básica, la de mejorar la calidad de su vida durante el tiempo que le queda, sea poco o mucho. Su propósito probablemente será lograr un mejoramiento psicológico a corto plazo -y esto, no porque, como dijo John Maynard Keynes, a largo plazo todos estaremos muertos.

Desde luego, muchos otros tipos de pacientes viven con una problemática muy semejante en este último respecto; pero las consecuencias distintivas para el tipo de paciente que estamos considerando aquí, provenientes de la penumbra perniciosa alrededor de su condicion, no se limitan a su falta de deseo de conseguir la legitimación de su papel de enfermo. Tal vez el paciente se haya acostumbrado por experiencias anteriores con otros médicos a la invasión, a veces brutal, de su privacidad, no sólo relacionado con exámenes clínicos y otras pruebas médicas, sino también en relación con interrogatorios sobre su vida sexual; tal vez el paciente ya esté acostumbrado al hecho de que diversos trabajadores de la salud lo vean como un bicho raro y al hecho de que su trato con él, por ejemplo, en relación con tomas de

${ }^{4}$ Cf. J. A. Izazola et al., "Conocimientos, actitudes y prácticas relacionadas con el SIDA: Bases para el diseño de programas educativos", en J. Sepúlveda Amor et al., sIDA, Cienciay Sociedad en México. México, 1989, pp. 297-336.

s Cf. Robert J. Levine, "AIDS and the physician-patient relationship", en Frederic G. Reamer (comp.), AIDS G Ethics. Nueva York, 1991, pp. 188-214. 
sangre, no sea igual a su trato con otros pacientes; pero lo que probablemente no habrá perdido es su preocupación por la confidencialidad de los asuntos tratados con los trabajadores de la salud.

\section{Confidencialidad y salud pública}

El juramento hipocrático impone la condición de confidencialidad a los médicos en sus relaciones con sus pacientes: "Io que en el tratamiento, o incluso fuera de él, viera u oyere en relación con la vida de los hombres, aquello que jamás deba divulgarse, lo callaré teniéndolo por secreto" ${ }^{6}$ Esta condición de confidencialidad es una constante histórica; el Código de Internación de Ética Médica de la Asociación Médica Mundial, por ejemplo, nos dice: "Un médico habrá de preservar el secreto absoluto de todo lo que oiga sobre su paciente en virtud de la confianza que se ha depositado en él".7 Hoy en día la ortodoxia predominante sobre la justificación de la imposición de esta condición de confidencialidad parecer ser la siguiente: si no se respeta en general esta condición, la gente por diversas razones tendrá pocas ganas de consultar a los médicos; si esto sucede, la consecuencia en el contexto de enfermedades como el SIDA, la hepatitis B, la tuberculosis y el cólera, será que muchas más personas correrán el riesgo de infectarse y de contraer la enfermiedad correspondiente.

Sobre esta ortodoxia hay que notar lo siguiente. Primero, depende de una cuestión empírica: la cuestión de si un debilitamiento de la condición de confidencialidad tendría de hecho el efecto postulado. En los casos de excepciones legales en los Estados Unidos a la condición de confidencialidad -heridas por arma de fuego, abuso de niños, enfermedades contagiosas- no se han encontrado pruebas significativas que apoyen la hipótesis de que tales excepciones legales hayan disminuido la propensión de los pacientes a buscar tratamiento y a cooperar en tal tratamiento. ${ }^{8}$

Que la cuestión empírica es crucial en este contexto es evidente en el conocido caso legal de Tarasoff $V$. The Regents of the University of California (1976). La decisión mayoritaria sostenía la obligación de un psiquiatra de advertir a una mujer que uno de sus pacientes estaba contemplan-

${ }^{6}$ Citado en D. Gracia, Fundamentos de Bioética. Madrid, 1989, p. 46.

${ }^{7}$ Citado en T. L. Beauchamp y J. F. Childress, Principles of Biomedical Ethics, 4 a. ed. Nueva York, 1994, p. 419.

${ }^{8}$ Cf. K. Appelbaum y P. S. Appelbaum, "The HIV antibody-positive patient", en J. C. Beck (comp.), Confidentiality versus the Duty to Protect: Foreseeble Harm in the Practice of Psychiatry. Washington, D. C., 1990, pp. 127-128. 
do asesinarla, sostenía "el deber de advertir" a terceras personas en riesgo de ser afectadas por sus pacientes; pero la opinión minoritaria, escrita por el Juez Clark, sostenía que "si no se asegura sólidamente la confidencialidad, aquellos que necesiten tratamiento se verán desanimados a buscar ayuda". Continuaba:9

\begin{abstract}
Sigue siendo un hecho desafortunado en nuestra sociedad que la gente que busca orientación psiquiátrica tienda a ser estigmatizada. Percatarse de tal estigma -cosa que se agudiza por la propensión de la gente que está en trance de considerar algún tratamiento a verse a sí misma desde el peor ángulo posible - crea una bien conocida resistencia a buscar ayuda. Esta resistencia disminuye cuando el psiquiatra asegura que guardará la confidencialidad.
\end{abstract}

Me parece que se necesita mucha más investigación empírica sobre este asunto; tomando en cuenta la referencia del Juez Clark a lo que podríamos llamar la propensión de ciertos pacientes a internalizar los estigmas sociales, tal investigación debe ser sensible a las diferencias entre enfermedades distintas -estigmatizadas/no-estigmatizadas, por ejemplo- y a las posibles diferencias culturales.

El segundo asunto sobre la justificación ortodoxa de la condición de confidencialidad es éste: aún si hubiera apoyo empírico para la presuposición tácita de esa justificación, esto solamente podría mostrar la deseabilidad de que la gente creyera que los trabajadores de la salud respetan tal condición. Mencionar esta posibilidad no es meramente una manifestación de cinismo de mi parte; hay razones para creer que se acerca a la realidad. El mismo Siegler, en otro trabajo, informa del descubrimiento de que el número de personas con acceso legítimo al expediente de uno de sus pacientes en su hospital era aproximadamente de setenta y cinco; ${ }^{10}$ otro estudio reveló que el $57 \%$ de médicos residentes había comentado sus casos con sus esposas y que el $70 \%$ de médicos residentes y estudiantes de medicina había comentado casos médicos en reuniones sociales. ${ }^{11}$ Como dijo el paciente de Siegler: "siempre creí que la confidencialidad médica era parte del código ético de los doctores. Tal vez debería usted decirme qué es exactamente lo que ustedes quieren decir con 'confidencialidad' "

${ }^{9}$ Citado en Beauchamp y Childress, op. cit., pp. 511-512.

${ }^{10} \mathrm{M}$. Siegler, "Confidentiality in medicine: a decrepit concept", en New England Journal of Medicine, núm. 307, 1982, pp. 1518-1521.

${ }^{1}$ B. D. Weiss, "Confidentiality expectations of patients, physicians, and medical students", en Journal of the American Medical Association, núm. 2247, 1982, pp. 2695-2697. 
Tercero: la ortodoxia hoy en día parece ser que la justificación de la confidencialidad se remite a ciertas consideraciones relativas a la salud pú. blica. Pero si esa fuera la justificación completa, tendría la consecuencia de que esta condición sólo estaría justificada en los casos de enfermedades trans. misibles. Esta no es la manera ortodoxa de entender el contenido de la condición de confidencialidad; por lo tanto o tenemos muchas confusiones sobre el asunto o la justificación de la condición tiene que remitirse a otras consideraciones adicionales. Se podría tratar de suavizar esta conclusión invocando la ignorancia de la gente sobre qué padecimientos son transmisibles; pero aparte de los casos en donde el nivel promedio de esta ignorancia es verdaderamente extraordinario, tal maniobra no salvará la ortodoxia frente a la dificultad señalada.

Queda un último punto en relación con la justificación ortodoxa. " $L a$ salud de mi paciente será mi primera prioridad", dice el Código de Ética Médica de la convención de Ginebra, ${ }^{12}$ capturando de esta manera la idea tradicional de una ética médica enfocada en el paciente. Muy diversas consideraciones han tenido como efecto el que esta "primera prioridad" puede en ciertas circunstancias estar en pugna con otras consideraciones que versan sobre el bienestar de la familia del paciente, sobre la salud de sus parejas sexuales y sobre la salud pública. Estos conflictos pueden surgir, pero aun cuando sea empíricamente cierto que han surgido, es una cuestión que está por decidir de qué manera precisa deben afectar tales conflictos a la práctica médica y a la relación médico-paciente. No es nada obvio que la mejor manera de resolver tales conflictos sea siempre mediante la modificación de aquella práctica y de esa relación. La aceptación razonable de la ortodoxia hoy en día sobre la confidencialidad requeriría que pensemos de nuevo sobre los valores involucrados en la práctica médica y en la relación médico-paciente. Las ortodoxias cambiantes no pueden ser sustitutos de tal pensamiento.

\section{Confidencialidad y el paciente individual}

Hay varias maneras aparentemente obvias de complementar la justificación ortodoxa de la imposición de la condición de confidencialidad; algunas podrían no solamente complementar esa justificación sino supuestamente tomar su lugar Primero, hay que recordar cómo se introdujo la justificación ortodoxa: si no se respeta en general la condición de confidencialidad, la gente se resistirá, por diversas razones, a consultar a los médicos. Ahora

\footnotetext{
${ }^{12}$ Citado en A. Campbell, G. Gillett y G. Jones, Practical Medical Ethics. Oxford, 1992, p. 160.
} 
bien, la idea entonces es que, dado el papel de los médicos, su función en términos de tratar de ayudar a la gente con problemas médicos sería en sí mismo un resultado poco deseable -sin importar cuál sea el tipo de problema médico en cuestión. Pero esta sugerencia comparte con la justificación ortodoxa su dudosa base empírica; además, presupone que el médico tiene la obligación de eliminar todos los estorbos para que la gente lo consulte a él. Aun cuando el médico tenga la obligación de tratar de ayudar a la gente que lo consulta, esta no equivale a la obligación de tratar de ayudar a toda la gente que podría consultarlo. En general, las obligaciones surgen dentro de las relaciones existentes, no dentro de meras relaciones posibles. ${ }^{13}$

Otra idea más prometedora es la siguiente: el respeto a la condición de confidencialidad, es, por lo menos en general, un prerrequisito para la obtención de la información necesaria para un diagnóstico adecuado. La idea es más prometedora, entre otras razones, porque la obligación postulada surge en este caso dentro de alguna relación existente.

Pero, supongo que todos los médicos del mundo están acostumbrados a escuchar muchas mentiras de sus pacientes ("¿cuánto fumas?", "¿cuánto bebes?") y también a enfrentar el fenómeno humano, tan humano, del autoengaño en esos mismos pacientes ("desde luego no soy homosexual", "claro que podría dejar de beber cuando me dé la gana", etcétera). Seguramente tales mentiras y tales estados de auto-engaño dificultan el diagnóstico; pero, como el caso de las compañias aseguradoras; lo ponen de manifiesto, no lo imposibilitan. Es decir, aun suponiendo que la falta de respeto a la condición de confidencialidad realmente aumentara la cantidad de mentiras y falseda. des que los médicos escuchan en la etapa previa al diagnóstico, no queda para nada claro cuáles serían las consecuencias empíricas de tal aumento en términos de diagnósticos subsecuentes equivocados. Y si no hay esas conse. cuencias adversas, sería algo extraño sostener que la base para justificar la "imposición" de la condición de confidencialidad es que tal "imposición" hace más cómodo el trabajo del médico.

Todas las supuestas justificaciones para imponer la condición de confidencialidad que hemos considerado hasta ahora han sido de índole consecuencialista; por esto se enfrentan de inmediato con las dudas que surgen debido a la relativa escasez de las correspondientes investigaciones empíricas. Una idea aparentemente muy distinta es la de derivar la condición de confidencialidad de un principio ético más general y más fundamental, el principio de autonomía o el principio de respeto hacia los individuos. Según Peter Singer, "el principio del respeto a la autonomía nos dice que

${ }^{13}$ Cf. Mark Platts, "Philosophical scepticism about moral obligation", en Proceedings of the Aristotelian Society Suplementary Volume, 1993, pp. 175-194. 
permitamos a los agentes racionales vivir sus propias vidas segun sus propias decisiones autónomas, libres de coerción o interferencias". ${ }^{14}$ Una formulación más detallada es la siguiente:

En el campo de lo ético, el concepto de autonomía significa que una persona tiene derecho, en principio, de acuerdo con sus propios valores y prioridades, a determinar aquello que debe suceder con su cuerpo o con su salud [...] El tratamiento de una persona como un fin en sí mismo y no como instrumento es un aspecto inherente al derecho a la dignidad humana, el derecho a ser respetado como persona.

La base moderna del concepto de autonomía se asienta en la concepción occidental del individuo como ser autónomo, capaz de dar forma y sentido a su vida [...] Del derecho a ser respetado como persona autónoma se desprende la obligación correlativa de los demás de no restringir [las elecciones intencionadas] de un individuo, hechas sobre la base de sus propios valores y prioridades, conciencia y creencias religiosas. ${ }^{15}$

Pero habrá que añadir, tal y. como lo hace el doctor Raanon Gillon, que este respeto a la autonomía "es la obligación moral de respetar la autonomía de los demás en la medida en que ese respeto sea compatible con un respeto igual a la autonomía de todos los potencialmente afectados". ${ }^{16}$

Supongo que el cambio dramático en las actitudes de los médicos norteamericanos en los últimos treinta años, su abandono de actitudes "paternalistas" con la mayoría de sus pacientes, podría interpretarse como su aceptación creciente del principio de autonomía. Así, en 1961 el 88\% de los médicos entrevistados indicó que habían tratado de evitar revelar un diagnóstico de cáncer al paciente, pero en 1 y/y, el $98 \%$ de los entrevistados informaron su política de decir la verdad al paciente con cáncer. ${ }^{17}$ Sin embargo, muchos otros factores, aparte de la aceptación creciente del princi-

${ }^{14}$ Peter Singer, Practical Ethics. Cambridge, 1979, p. 142.

is $\mathrm{H}$. Fuenzalida-Puelma et al., "Normatividad sobre sIDA en América Latina y el Caribe", en Fuenzalida-Puelma et al. (comps.), Aportes de La Etica y el Derecho al Estudio del SIDA. Washington, D. C., 1991, p. 32.

${ }^{16}$ Raanon Gillon, "Preface: Medical ethics and the four principles", en R. Gillon (comp.), p. .xii.

"Cf. D. Oken, "What to tell cancer patients: a study of medical attitudes", en Journal of the American Medical Association, núm. 175, 1961, pp. 1120-28 y D. H. Novack et al., "Changes in physicians attitudes toward telling the cancer patient", en Joumal of the American Medical Association, núm. 241, 1979, pp.897-900. 
pio de autonomía, podrían haber producido este tipo de cambio. Supongo también que algunas investigaciones sobre las actitudes de los pacientes podrían interpretarse como si apoyaran la atribución a ellos del deseo de que los médicos respeten su autonomía. Por ejemplo, en una investigación hecha a treinta pacientes psiquiátricos en relación con preguntas hipotéticas sobre confidencialidad, el $\mathbf{8 0 \%}$ indicó que si se les asegurara guardar confidencialidad, su relación con el equipo médico mejoraría, el $67 \%$ dijo que se molestarían o enojarían si se hiciera pública sin su consentimiento la información verbal que han dado, el $17 \%$ indicó que abandonaría el tratamiento si esto sucediera, y el $95 \%$ dijo que les molestaría que sus expedientes fueran mostrados a otros sin su consentimiento. ${ }^{18}$ Una investigación posterior sobre 58 pacientes psiquiátricos de consulta externa reveló que todos dijeron que reaccionarian fuertemente, quejándose y en algunos casos interponiendo acción legal, si ocurrieran faltas de respeto a la confidencialidad. ${ }^{19}$ (Desde luego, tales aseveraciones no son predicciones confiables; pero aunque así fueran, no apoyarían la justificación ortodoxa de la confidencialidad en términos de la resistencia de potenciales pacientes a buscar ayuda médica.) Pero, de nuevo, muchos otros factores, aparte del deseo o la expectativa de que los médicos respeten su autonomía, podrían haber producido las actitudes expresadas por los pacientes.

Es evidente que hay cierta oscuridad tanto en el contenido como en la justificación del principio de autonomía; en consecuencia hay cierta oscuridad también sobre la manera como este principio apoya la imposición de la condición de confidencialidad en las relaciones entre los médicos y sus pacientes. Pero es más importante señalar otra dificultad que enfrenta esta propuesta. Tal como la presenté, una motivación para esta propuesta fue la de evitar las dificultades que enfrentan los otros intentos de justificación debido a su naturaleza consecuencialista. Pero hay que recordar las palabras de Gillon: el respeto a la autonomía "es la obligación moral de respetar la autonomía de los demás en la medida en que ese respeto sea compatible con respeto igual a la autonomía de todos los potencialmente afectados". El respeto a la autonomía del paciente Prosenjit Poddar resultó no ser compatible con el respeto a la autonomía de Tatiana Tarasoff. Así resultó; pero en tanto que guía para la acción, el principio de autonomía presupone que podemos calcular las probables consecuencias del respeto a la autonomía

${ }^{18} \mathrm{Cf}$. D. Schimd et al., "Confidentiality in psychiatry: a study of the patients view", en Hospitaland Community Psychiatry, núm. 34, 1983, pp. 353-355.

${ }^{19} \mathrm{Cf}$. P.S. Appelbaum et al., "Confidentiality: an empirical test of the utilitarian perspective", en Bulletin of the American Academy of Psychiatry and the Law, num. 12, 1984, pp. 109-116. 
de una persona en términos de la autonomía de otras personas: un cálculo que, no obstante la diferencia en terminología, no dista mucho de los cálculos problemáticos involucrados en los otros intentos de justificación de la condición de confidencialidad. Si no respetamos la autonomía de Poddar, por ejemplo, ¿cuáles serán las consecuencias para la autonomía de otros potenciales pacientes psiquiátricos que tal vez como consecuencia no buscarán ayuda médica? Aún suponiendo que el principio de autonomía implica alguna condición de confidencialidad, no podemos saber exactamente qué condición hasta no tener información empírica confiable sobre las probables consecuencias de la aceptación de cada una de las condiciones propuestas como candidatos.

\section{Confidencialidad y confianza}

Uno puede respetar o no la autonomía de un desconocido. Un médico que sigue los lineamientos emitidos en 1985 por el General Medical Council del Reino Unido ni siquiera tiene que tener contacto con una persona en riesgo en el proceso de advertirle del peligro: puede meramente comunicar los hechos pertinentes al médico que tiene a su cargo a esa persona. ${ }^{20}$ Por contraste, la última idea sobre la justificación de la confidencialidad que quisiera examinar se centra en el hecho de que el médico y el paciente sí se conocen y en el hecho de que la meta tentativa detrás de este conocimiento mutuo es la de llegar a consolidar una relación plena médico-paciente, una firme amistad médica basada en la existencia de un profundo vínculo de confianza entre el paciente y el médico.

Este tipo de confianza presupone por ambas partes la creencia de que uno no hará nada para dañar intencionalmente al otro. Ahora bien, es evidente que algunas faltas de confidencialidad pueden ocasionar un daño considerable. Cuando el doctor William Behringer resultó ser seropositivo por el ViH en el centro médico donde trabajaba en New Jersey, recibió en pocas horas llamadas telefónicas de apoyo de miembros del personal médico del centro; en pocos días recibió llamadas semejantes de sus pacientes del mismo centro; poco después su práctica médica estaba absolutamente en ruinas. ${ }^{21}$ Dudo que una experiencia semejante pudiera ayudar a la consolidación de una relación plena médico-paciente.

${ }^{20} \mathrm{Cf}$. A. Orr. "The legal implications of AIDSand HIV infection in Britain and the United States", en B. Almond (comp.), AIDs: A Moral Issue. Nueva York, 1990, pp. 112-139.

${ }^{21}$ Superior Court of New Jersey, Law Division, Mercer County, Docket, núm. 1882550, 25 de abril de 1991 . 
Quizás esta sea la idea detrás de la razón que el Código Internacional de Ética Médica ofrece en apoyo de la condición de confidencialidad: "Un médico habrá de preservar el secreto absoluto de todo lo que oiga sobre su paciente en virtud de la confianza que se ha depositado en él". Pero aun cuando cualquier relación de confianza presuponga cierto grado de confidencialidad, en el caso de una relación médico-paciente del tipo deseado este grado aumenta por una razón evidente. El paciente voluntariamente sacrifica casi toda su privacidad, permite que se le hagan preguntas sobre aspectos íntimos de su vida que difícilmente le permitiría hacer a cualquier otra persona; a cambio de este sacrificio su exigencia es de pleno respeto a la confidencialidad en relación con sus contestaciones a tales preguntas. Además, en el caso de pacientes psiquiátricos (y algunos otros) esta exigencia se verá aumentada aún más por la propensión mencionada por el Juez Clark a internalizar la estigmatización social de su condición; en este contexto, las aseveraciones antes mencionadas de los pacientes psiquiátricos respecto de las faltas de confidencialidad no son nada sorprendentes.

Lo que sí es algo sorprendente es el hecho de que los filósofos morales hayan prestado tan poca atención al concepto de confianza; una consecuencia inmediata de esto es que el fundamento de esta última justificación de la condición de confidencialidad no es nada transparente. Hay que reconocer también que este tipo de justificación de tal imposición sólo podría funcionar completamente dentro de estructuras médicas diseñadas para hacer más probable el surgimiento de relaciones plenas entre médicos y pacientes; tal justificación se debilita considerablemente, por ejemplo, en contextos en donde hay frecuentes cambios en el médico que atiende a un mismo paciente. Con todo, sospecho que esta idea sobre la confidencialidad captura un elemento básico en el pensamiento de mucha gente sobre este asunto, tanto en médicos como en pacientes. Las reacciones de pacientes frente a las violaciones de la mera privacidad -por ejemplo, cuando algún desconocido consigue tener acceso a sus expedientes- son, sospecho, totalmente distintas a sus reacciones frente a abusos de confidencialidad por parte de los médicos que los conocen personalmente; pero se necesita hacer investiga. ciones empíricas sobre esta cuestión, aparte de mucho más trabajo filosófico sobre los conceptos evaluativos involucrados en ella.

\section{Confidencialidad en la práctica}

Una justificación de la condición de confidencialidad no tiene por que ceder a la tentación -característicamente filosófica pero no exclusivamente de filósofos- de buscar una teoría monolítica de tal condición: la justificación 
podría ser una teoría híbrida que combinara varias ideas. Tampoco habrá que esperar de tal justificación una fórmula mecánica para resolver los casos de conflictos entre la obligación de confidencialidad y otras consideraciones morales: hay verdaderos dilemas morales. De hecho las preguntas prácticas sobre la confidencialidad no suelen ser en general demasiado dificiles. Supongamos que nuestro paciente homosexual y seropositivo expresa a su psicoanalista su intención de tener relaciones sexuales de alto riesgo con gente desconocida. Por lo anónimo de las parejas potenciales, hay poco que el psicoanalista pueda hacer, aparte de advertirle al paciente del riesgo que representaria $s u$ salud una reinfección por el VIH o una infección por otras enfermedades sexualmente transmisibles y aparte de hablar con él sobre su enorme responsabilidad moral de no infectar a otras personas. En todo caso el psicoanalista podría tratar de convencer al paciente de lo conveniente y lo moral del uso del condón. Pero si el mismo paciente expresa al psicoanalista su intención de ocultar su seropositividad a su pareja estable y de seguir teniendo relaciones sexuales con ella sin ningún tipo de protección, entonces surgen nuevas posibilidades de acción por parte del psicoanalista además de las ya mencionadas. Primero, podría recordarle al paciente cómo cambiaría el tipo de relación que tiene actualmente: la relación que evidentemente le importa se vería deteriorada por tener a la base una falta total de consideración hacia la otra persona involucrada. ${ }^{22} \mathrm{Si}$ esto no tiene el efecto deseado, habrá también la posibilidad de advertir a la persona que se encuentra en riesgo, aun cuando esta acción del psicoanalista pudiera acabar con cualquier posibilidad de desarrollo de una relación plena entre este médico y este paciente. Pero en este respecto la situación no es moralmente tan diferente de otras en las que llegan a estar en pugna consideraciones surgidas de otros tipos de amistades potenciales -sean "institucionalizadas" o no- con consideraciones sobre el bienestar de otras personas conocidas.

Seguramente hay médicos para quienes las preguntas prácticas sobre la confidencialidad en relación con el tipo de paciente bajo consideración pueden volverse más difíciles: en situaciones, por ejemplo, en donde las relaciones homosexuales ejemplificadas sean ilegales o en donde simplemente exista una regla de notificación obligatoria para todo médico que diagnostica una infección por el viH junto con un sistema de rastreo de los contactos sexuales previos de los pacientes. Pero mi interés aquí en considerar el asunto de la confidencialidad ha sido otro, menos directamente práctico. Si la última idea mencionada sobre la justificación de la condición de confidencialidad es, por lo menos en ciertos contextos, más o menos correcta, tiene como consecuencia que esta condición alcanzará una importancia característica

${ }^{22} C f$. Campbell, Gillett y Jones, op. cit., p. 126. 
en los casos de pacientes seropositivos y homosexuales y en búsqueda de ayuda psicoanalítica dentro de la actual situación en México: éste es un síntoma importante de la peculiaridad de la relación entre este tipo de paciente y este tipo de médico en tal situación.

\section{¿Hacia la relación plena?}

He mencionado por lo menos un factor que pudiera surgir en la etapa de acomodo entre el paciente y el médico que claramente podría tener la consecuencia de que la relación médico-paciente terminara en ese momento: la determinación del paciente de poner en riesgo la salud de su pareja estable y la consecuente obligación por parte del médico de advertirie a esa pareja del riesgo que corre. Es también posible que para muchos médicos el otro caso mencionado, la determinación del paciente de tener relaciones sexuales de alto riesgo con gente desconocida, sería un caso en donde también habría razones suficientes para poner fin a la relación profesional. Pero muchas otras consideraciones podrían tener consecuencias semejantes. El psicoanalista podría sencillamente dudar de su propia competencia para ayudar a este tipo de paciente: ya hemos señalado que la específica combinación de homosexualidad más seropositividad ha recibido muy poca atención por parte de los psicoanalistas. Podría también dudar, dentro de los términos de su teoría psicoanalítica, de la capacidad psíquica de este potencial paciente para manejar con éxito el proceso de su psicoanálisis. Podría creer que no existe el acuerdo necesario entre médico y paciente sobre las metas y los medios del tratamiento: tal vez el paciente esté buscando "curarse" de su condición de homosexual pero el médico no acepta tal meta. El psicoanalista podńa tener, conscientemente o no, una profunda aversión a pacientes homosexuales: en el caso en que esta aversión fuera consciente, esto podría conducirto a reflexionar sobre la poca probabilidad de lograr establecer con el paciente una relación plena médico-paciente. Podría tener miedo plenamente irracional de atrapar la infección por el viH como consecuencia del tratamiento: es común que los médicos que sí tratan a pacientes seropositivos o con SIDA reconozcan que tienen este miedo irracional. ${ }^{23}$ (Es dificil saber si el hablar de "peste", confundiendo así lo contagioso con lo infeccioso, sea causa o efecto de tal miedo.) Podría tener miedo acerca de las consecuencias para su práctica médica si se llegara a saber que tiene pacientes de este tipo: probablemente la penumbra perniciosa de actitudes negativas sobre la con-

${ }^{23}$ Cf. G. Friedland, "Clinical care in the AlDs epidemic", en Daedalus, núm. 188, 1989, pp. 59-83. 
dición de este paciente ha invadido a otros pacientes suyos y a otros pacientes potenciales. En el caso en que el paciente desarrolle la enfermedad del SIDA, el psicoanalista podría dudar de su propia capacidad para soportar los efectos de esa misma penumbra perniciosa de actitudes negativas por parte de los familiares y los "amigos" del paciente.

Dos posibles consideraciones que pueden llevar a un psicoanalista a tomar la determinación de no aceptar el tipo de paciente en cuestión merecen mención aparte -aun cuando ambos pudieran estar relacionados con algunos de los factores que acabamos de mencionar. Una, es que el psicoanalista podría tener, como cualquier otro médico, una fuerte aversión a los pacientes con condiciones médicas potencialmente mortales. En sus estudios clásicos sobre el proceso de morir, Elisabeth Kübler-Ross distingue cinco etapas del morir en pacientes que reconocen su condición médica potencialmente mortal: negación y aislamiento, enojo, etapa de negociación, de. presión y aceptación. ${ }^{24}$ (Desde luego, este modelo es igualmente flexible que el modelo de Siegler de la relación médico-paciente.) Kübler-Ross explícitamente extendió el uso de este modelo para describir las reacciones emocionales de los familiares de los pacientes. Muchos otros han extendido el uso de este modelo para describir las reacciones de muchas sociedades frente a la problemática del SIDA, y supongo que hay otros que quieren emplearlo en la descripción de las reacciones emocionales de la gente homosexual frente al fenómeno de su homosexualidad. Lo que es menos reconocido es el hecho de que la misma Kübler-Ross también explícitamente extendió el uso de este modelo para describir las reacciones emocionales de muchos trabajadores de la salud frente al proceso del morir de sus pacientes. ${ }^{25} \mathrm{Si}$, en este contexto, un psicoanalista duda de su propia capacidad de manejar sus reacciones emocionales y en consecuencia decıe no aceptar ningún paciente con algún padecimiento potencialmente mortal, me parece que estaría en todo su derecho (cosa que no implica que por esto será más simpático).

La segunda posible consideración que quisiera enfatizar es la siguiente: desde hace muchos años se ha desarrollado dentro de algunas teorías psicoanalíticas un examen del fenómeno de la contratransferencia, entendida ésta como una reacción emocional del psicoanalista frente al paciente.

[La contratransferencia] es lo que siente [el psicoanalista] hacia sus pacientes en la psicoterapia psicoanalítica -ya sea individual o grupal-, producto de su historia, complejos, cultura, aparato psí.

${ }^{24}$ Cf. Elisabeth Kübler-Ross, On Death and Dying. Nueva York, 1969.

${ }^{25}$ Cf. E. Kübler-Ross, AIDs: The Ultimate Challenge. Nueva York, 1987, p. 2. 
quico, etcétera, también de lo que el paciente le hace sentir con su personalidad y su transferencia. ${ }^{26}$

Ahora bien, estas teorías psicoanalíticas consideran el fenómeno de la contratransferencia útil en general tanto para el psicoanalista como para el paciente; pero si un psicoanalista duda de su propia capacidad de manejar sus reacciones contratransferenciales en el caso de un potencial paciente específico y por lo tanto decide no aceptar a ese paciente, también me parece que estaría en todo su derecho (cosa que no implica que por esto sería más admirable).

\section{¿Una nueva relación plena?}

Se ha dicho que los fenómenos del VIH y el SIDA han dado lugar a la demanda por parte de los pacientes de una nueva relación medico-paciente; esa demanda ha tenido como consecuencia -entre otras cosas- que los pacientes ya no son tan pacientes. Plantear tal demanda parece presuponer -cosa que está muy lejos de ser el caso- que tenemos un entendimiento adecuado de lo que sería realmente una relación plena entre médicos y pacientes. Este tema requeriría de un tratamiento mucho más detallado de lo que puedo ofrecer aquí; así, para terminar ofreceré solamente algunos comentarios muy breves sobre el asunto.

Dije antes que se necesita mucho más trabajo conceptual sobre la noción clave de confianza; no le asombrará a nadie si añado ahora que también se necesita mucho más trabajo empírico sobre, por ejemplo, las distintas maneras como de hecho conceptualizan la relación plena tanto los médicos $\mathrm{y}$ otros trabajadores de la salud como los pacientes y sus familiares. Es verdad que hay algunos documentos personales de gran valor sobre esta cuestión: el libro de Susan Sontag acerca de su experiencia como paciente, ${ }^{27}$ la extraordinaria descripción que nos ofrece John Berger de la vida del médico John Sassall dentro de un pequeño poblado pobre del norte de Inglaterra, ${ }^{28}$ el relato angustioso de la psicoanalista Luisa Rossi de su tratamiento de pacientes homosexuales y seropositivos. ${ }^{29}$ También es verdad que hay algunas investigaciones empíricas más sistemáticas sobre algunas cuestiones perti-

${ }^{26}$ J. de J. González Núñez, La Fortaleza del Psicoterapeuta: La Contratransferencia. México, 1989, p. 11.

${ }^{27}$ Susan Sontag, Illness as Metaphor. Nueva York, 1978.

${ }^{28} \mathrm{John}$ Berger, A Fortunate Man: The Story of a Country Doctor. Londres, 1967.

${ }^{29}$ Luisa Rossi, Un espejo frente al otro. México (en prensa). 
nentes para el tema que nos interesa. ${ }^{30}$ Pero aún así, nos queda todavía mucho trabajo que hacer para iniciar una reflexión razonable acerca de las discrepancias sobre asuntos valorativos involucradas en las distintas maneras de conceptualizar la relación plena médico-paciente. Y, desde luego, el desarrollo de las investigaciones empíricas requeridas debe ser sensible a la diversidad de contextos donde se ubican las relaciones entre médicos y pacientes: la problemática de un psicoanalista al inicio de un tratamiento con un paciente homosexual y seropositivo no podría estar más alejada de la problemática de otro médico en el servicio de urgencias de un hospital público en México que tiene frente a sí un paciente con SIDA en inminente riesgo de morir.

${ }^{30}$ Por ejemplo, Weiss, op. cit.; Strull, W.M., et al., "Do Patients want to participate in medical decision making?", en Journal of the American Medical Association, num. 252 , 1984, pp. 2990-2994; S. H. Miles, "Informed demand for 'non-beneficial' medical treatment", en New England Journal of Medicine, núm. 325, 1991, pp. 512.515. 